\title{
Correlation and Wear-Time Compliance of the Wrist-Worn SQORD Activity Monitor Compared to the Actigraph 3TGX in Measuring Free-Living Physical Activity in Low SES Elementary Youth
}

\author{
Kelsey McAlister (D) ${ }^{1}$, Koren Fisher (iD) ${ }^{1}$, Kathleen Wilson (iD ${ }^{1}$, and Risto Marttinen (iD) 2 \\ ${ }^{1}$ California State University, Fullerton, Department of Kinesiology \\ ${ }^{2}$ George Mason University, College of Education and Human Development
}

\begin{abstract}
Background and Purpose: Activity trackers have grown increasingly popular, yet research grade accelerometers, like Actigraph, are still very expensive and must be worn on the waist. This study correlated the low-cost wrist-worn SQORD band to the Actigraph accelerometer to assess physical activity (PA) in youth from a low-income area. Methods: Forty-one participants (22:19 male:female; mean age $=10.7$ years) in an after-school program participated. Participants wore a waist-worn Actigraph accelerometer and a wrist-worn SQORD for seven days. Abdominal, upper and lower body strength and endurance, and aerobic capacity were assessed through FITNESSGRAM tests. Bivariate correlations were used to analyze the relationship between moderate-to-vigorous PA (MVPA) via the Actigraph and SQORD and to examine relationships between physical fitness and MVPA. Results: At 8- and 10-hour wear-time, compliance with wearing the devices was higher with the SQORD than the Actigraph. A correlation was found between MVPA via SQORD and Actigraph $(r=0.651)$. Neither the SQORD or Actigraph was associated with any fitness measures $(r=-0.061 ;-0.817)$. Conclusion: The SQORD appears to be successful in tracking MVPA in youth and was worn more than the Actigraph. Wrist-worn, consumer-grade devices may be a cost-effective alternative to traditional accelerometers for physical education programs and research in lowincome populations.
\end{abstract}

(C) 2019 and CC-BY 4.0 licensed by the authors.

Keywords: activity devices, children, low-income, SQORD band

\section{Introduction}

The Centers for Disease Control and Prevention (CDC) recommends youth aged 6-17 years participate in at least 60 minutes of moderate-tovigorous physical activity (MVPA) a day (CDC, 2017). Despite these guidelines, a large proportion of youth are not sufficiently physically active. Estimates suggest just $22 \%$ of youth meet the recommended MVPA guidelines, highlighting the need to increase physical activity in this population (Katzmarzyk et al., 2016). Andersen and colleagues (2006) have shown that one hour of MVPA daily is associated with reduced likelihood of cardiovascular risk factors and improved body composition in youth. Beyond physiological outcomes, benefits of MVPA for youth also include educational outcomes such as cognition, connectedness, and a reduction in absenteeism and dropout rates (Andersen et al., 2006; Basch, 2011).

Low participation in MVPA has been associated with several sociodemographic characteristics. Rates of MVPA participation are lower in Black and Hispanic youth than in White, with these disparities especially evident in females (Basch, 2011). Brodersen and colleagues (2007) found that Black youth spent more time participating in sedentary behaviors than White youth. This same study also found youth from lower socioeconomic status (SES) families participated in sedentary behavior 2.29 and 4.09 hours more per week, respectively, compared to youth from higher SES families. A recent systematic review by Stalsberg and Pedersen (2010) found that 58\% of 62 studies reported that youth ages 13 to 18 
years with higher SES were more active than youth with lower SES. Parental education level also appears to be associated with youth MVPA levels. Frederick, Snellman, and Putnam (2014) showed that $11-13 \%$ more of youth with collegeeducated parents reported being physically active than youth whose parents held only a high school diploma. For youth of low SES, school-based MVPA opportunities may be an important point of intervention given that those who participate in the lowest levels of MVPA have been shown to have the least access to school-based activity opportunities and resources (Basch, 2011).

With youth MVPA participation rates so low, strategies to promote an active lifestyle among those aged 6 to 19 are essential, especially in lowincome areas. Mobile devices and fitness trackers have become increasingly popular methods for raising self-awareness regarding physical activity behavior (Case, Burwick, Volpp, \& Patel, 2015; Fanning, Mullen, \& McAuley, 2012). New gamebased technologies have been promoted as a method for increasing MVPA in obese youth (Garde et al., 2015). Recent studies have shown that consumer-grade devices are valid for measuring physical activity in adults (Dominick, Winfree, Pohlig, \& Papas, 2016; Evenson, Goto, \& Furberg, 2015; Ferguson, Rowlands, Olds, \& Maher, 2015). However, there is limited evidence regarding appropriate and effective fitness tracking technology for youth in a low-income school/after-school setting.

Research-grade accelerometers have been widely used in large-scale, national studies, and have also been used to establish validity of other consumer-based fitness tracking devices (e.g. Fitbit Flex and Fitbit Zip) in a number of studies (Chu et al., 2017; Lee, Williams, Brown, \& Laurson, 2014; Tully, McBride, Heron, \& Hunter, 2014). Although the Actigraph GT3X accelerometer (Actigraph Corporation, 2017) is used extensively in research, it is not an appropriate activity-tracking tool for the consumer, especially for youth, due to its lack of simple MVPA feedback (i.e., the Actigraph does not have a display and the software must be purchased to view MVPA levels).
Less is known about consumer-grade activity monitors that have been developed specifically for use in youth populations, especially in comparison to research-grade accelerometers. The SQORD band is a wrist-worn device that measures the duration and intensity of physical activity, linking the data to an online platform and the user's mobile phone, where participants can create an avatar of themselves and participate in a game. The SQORD band was designed with youth in mind, is considerably less expensive than research-grade accelerometers, and syncs wirelessly to the user's mobile phone, providing direct feedback regarding activity (SQORD, 2015).

One method to better understand activity monitors, including accelerometers, is by relating physical activity captured by the devices to performance on measures of fitness. Although physical activity and fitness level are two different constructs, there is evidence showing that higher levels of physical activity are correlated with higher fitness levels in youth (Aires et al., 2010; Lohman et al., 2008; Martinez-Gomez et al., 2011). Cardiorespiratory and muscular fitness levels have both been shown to be associated with physical activity among adolescents (Aires et al., 2010; Lohman et al., 2008; Martinez-Gomez et al., 2011). Although previous studies have demonstrated relationships between daily physical activity measured by accelerometry and fitness level, these associations have yet to be established using the SQORD band.

As outlined earlier, MVPA has previously shown to be inversely associated with SES; therefore, finding methods to increase physical activity in low SES populations is especially important. The SQORD band is a cost- and consumer-friendly device that tracks physical activity in youth and provides quick, simple feedback for the user; however, data regarding the associations of the SQORD band's output compared to a researchgrade accelerometer in a low-SES population is lacking. To our knowledge, only two studies have examined the SQORD band and to date (Masteller, Sirard, \& Freedson, 2017; Sirard, Masteller, Freedson, Mendoza, \& Hickey, 2017), there are no published data on the wear-time 
necessary to obtain accurate MVPA data using the wrist-worn SQORD band or if a low SES population would prefer the SQORD band compared to a research-grade accelerometer. The SQORD band also reports activity in the form of arbitrary points (e.g., instead of 10,000 steps the SQORD band will say a person gained 90,500pts), which has yet to be correlated with a specific level of physical activity. Thus, the purpose of this study was to assess daily MVPA and differences in wear-time compliance recorded by a consumer-grade, wrist-worn activity monitor (SQORD band) in comparison to a research-grade, waist-worn accelerometer (Actigraph accelerometer). This study focused on elementary school-aged youth from low-income schools. The relationships between daily MVPA measured by the SQORD band, cardiorespiratory fitness, and muscular fitness were examined in order to better understand the SQORD band. We hypothesized that the SQORD band would correlate highly with the Actigraph due to past wrist-worn activity monitors (e.g., Fitbit) showing strong correlations to the Actigraph (Chu et al., 2017; Lee, Williams, Brown, \& Laurson, 2014; Tully, McBride, Heron, \& Hunter, 2014).

\section{Methods}

\section{Study Design}

Data were drawn for this quantitative study are from a baseline measure of a larger intervention study examining the effects of an after-school physical activity program on literacy and fitness called Reflective Educational Approach to Character and Health (REACH) (see Marttinen \& Fredrick, 2017; Marttinen et al., 2019 for a detailed description of REACH). Participants were given both a waist-worn research-grade Actigraph accelerometer and a wrist-worn lowcost SQORD accelerometer. Due to availability of devices, not all participants received both devices as the number of Actigraph accelerometers available to the study were limited. Thus, a portion of the cohort (27 participants) was randomly selected to receive both devices, with the remaining participants receiving only the SQORD band.

\section{Participants}

Data from a total of 41 fifth and sixth grade students were analyzed. Students average age was $10.7 \pm 0.7$ years. The population selfidentified $54 \%$ male, $46 \%$ female; $71 \%$ were in $6^{\text {th }}$ grade and the students self-identified $80.5 \%$ Hispanic; $7.5 \%$ White; $5 \%$ Black, $7.5 \%$ other.

\section{School Demographics}

The school where the study was conducted is a Title I school and qualifies for supplemental government funding due to the high percentage of students coming from low-income families. There were 757 students enrolled during the 2016-2017 school year, of which $623(82.2 \%)$ were receiving either free or reduced-price lunches. The majority of the students $(87 \%)$ were Latina/o (Education Data Partnership, 2017).

\section{Physical Activity Measures via Actigraph Accelerometry}

Daily MVPA was measured using Actigraph triaxial accelerometers (Actigraph 4 MB GT3X, Penscacola, FL). These devices provide detailed information regarding temporal patterns of physical activity, including duration, frequency, and intensity. All accelerometer data were collected and stored as acceleration counts, recorded in 5-second epochs. The Actigraph triaxial accelerometer has excellent inter- and intrainstrument reliability across a wide range of accelerations and has been validated for children ages 10 to 15 years (Hänggi, Phillips, \& Rowlands, 2013).

Accelerometers were distributed to students during an after-school program by research staff. Participants were instructed to wear the accelerometer around their waist on the right side for as much of the day as possible (excluding sleeping, showering/bathing, and swimming activities) for seven consecutive days. Each participant was given instructions as to how the accelerometer works, how to put on and remove the device, and how to adjust the elastic waistband. Participants were also provided a handout to share with their parents describing proper wear of accelerometers in either English or Spanish. At the end of the seven-day period, the accelerometers were collected by research staff at the same after-school program. 
The accelerometer data were screened for weartime compliance, where valid wear-time criterion was a priori defined as at least 10 hours per day for a minimum of four days, including at least one weekend day, after excluding the first and last day of data (Rich et al., 2013). Data from the first and last days were excluded because they coincided with the days that the accelerometers were given out and collected, respectively, and therefore were incomplete days of wear. Preliminary analyses revealed there was very low compliance with the a priori wear-time criterion of 10 hours per day. The wear-time criterion was therefore reset at a minimum of 6 hours per day to optimize the sample size. Non-wear time was defined as any period of 60 minutes or more of zero accelerometer counts per minute (cpm). Thresholds established specifically for children by Evenson et al. (2008) were used to determine time spent in physical activity at all intensities. MVPA was determined by the sum of time spent in moderate $(\geq 2296-<4012 \mathrm{cpm})$ and vigorous physical activity $(\geq 4012 \mathrm{cpm})$.

\section{Physical Activity Measures via SQORD}

Daily MVPA was also measured using an activity tracker designed for youth called the SQORD band. The SQORD band is a waterproof, wristworn, non-display device and has only been used in research twice thus far (Masteller et al., 2017; Sirard et al., 2017). The SQORD band measures physical activity as arbitrary counts in 15-minute blocks. These arbitrary counts, or "points," then sync to the participant's mobile device, in which the participant can play a game based off of how many points they have earned. The SQORD band can save and store earned activity points for seven days. A greater number of points earned in a 15minute period is indicative of a higher level of intensity of physical activity. These scores are based off of a proprietary algorithm that SQORD uses to convert the intensity of steps per minute to points in order to engage the user with "SQORD points."

The SQORD band was distributed to participants at the same time as the Actigraph accelerometers. Participants were instructed to wear the SQORD band for the entire seven-day physical activity data collection period while also wearing the
Actigraph accelerometer. Following the data collection period, the activity data from the SQORD was screened for wear-time. Valid weartime was established as 6 hours per day, on at least 4 days including 1 weekday and 1 weekend day, so as to match the wear-time criterion established for the Actigraph data. For the SQORD band, valid days were defined as a minimum of 24 15-minute blocks where points were accumulated. The first and the last days were excluded, as these were the days the SQORD band was given out and returned, respectively. Average daily SQORD points were calculated for each participant meeting the weartime criterion.

\section{Fitness Measures}

Physical fitness in four areas (abdominal, upper and lower body strength and endurance, and aerobic capacity) was assessed in all participants using four separate tests, as described below. All tests other than the lower body power test were performed using the FITNESSGRAM battery of tests (Plowman, 2013). The FITNESSGRAM is a validated and reliable test for fitness in youth populations and has been adopted by several states as a standardized test including California where this study took place.

\begin{abstract}
Abdominal Strength and Endurance Assessment. A cadence-based curl-up test, following the FITNESSGRAM standards, was used to obtain abdominal strength and endurance (Plowman, 2013). Participants were instructed to complete a curl-up to the rhythm of a metronome. Participants began the test lying on their back, with the knees bent, and feet unanchored. The metronome was set to a cadence of 20 repetitions per minute. Participants were asked to complete as many curl-ups as possible until they felt they could no longer continue, with 75 curl-ups being the maximum (Plowman, 2013).
\end{abstract}

This type of curl-up test has been shown to have lower reliability in younger participants compared to older, but this is consistently found with abdominal assessments (Anderson, Zhang, Rudisill, \& Gaa, 1997; Plowman, 2013). Testretest reliability for the curl-up test has previously been reported to be 0.70 in youth 6-10 years old and $0.86-0.89$ in youth aged 10-12 years old, 
respectively (Anderson et al., 1997; Patterson, Rethwisch, \& Wiksten, 1997).

Upper Body Strength and Endurance Assessment. The $90^{\circ}$ push-up assessment, following the FITNESSGRAM standards, was used to obtain upper body strength and endurance, specifically upper arm and shoulder girdle (Plowman, 2013).

The $90^{\circ}$ push-up test was conducted at a cadence of one repetition per every three seconds. Participants placed hands and toes on the grounds, without contact between the knees and the ground, and were instructed to flex their arms to $90^{\circ}$ and then push back up. Participants were asked to complete as many push-ups to the beat of the metronome until they could no longer continue (Plowman, 2013). This test has demonstrated reliability coefficients ranging from 0.64 to 0.75 in elementary-aged youth (Plowman, 2013; Saint Romain \& Mahar, 2001).

Aerobic Capacity. The PACER test was used to measure estimated maximal oxygen consumption $\left(\mathrm{VO}_{2 \max }\right)$. The PACER test is a multistage test adapted from the 20-meter shuttle run test and was administered following the FITNESSGRAM protocol. Each participant was instructed to run back and forth across a course that was 20 meters in length. Beeps on a soundtrack played throughout the test, with each beep indicating when a participant should reach the ends of the course. As the test proceeded, the pace of the beeps was progressively increased after each minute until the participant could no longer maintain the set pace (Plowman, 2013). An equation developed by Mahar et al. (2011) was used to estimate $\mathrm{VO}_{2 \max }$ from the results accounting for age and sex. Results are presented as an estimate of $\mathrm{VO}_{2 \max }$ in $\mathrm{ml} \cdot \mathrm{kg}^{-1} \cdot \mathrm{min}^{-1}$. The PACER test has been found to be both valid and reliable, with several studies reporting validity coefficients ranging from 0.83 to 0.93 and reliability coefficients above 0.64 (Leger \& Gadoury, 1989; Leger \& Lambert, 1982; Leger, Mercier, Gadoury, \& Lambert, 1988; Paliczka, Nichols, \& Boreham, 1987; Ramsbottom, Brewer, \& Williams, 1988).
Leg Power. Leg power was assessed using the vertical jump test (DiStefano et al., 2010). Vertical jump height was measured using a vertical jump apparatus (Epic Combine Jump Station), with each vane of the apparatus representing 0.5 inches of jump height. Participants attempted two practice countermovement jumps at a self-selected depth before performing three maximal jumps. If the participant touched any vanes on the third attempt, they continued to jump until they no longer moved any vanes. The best trial was recorded and used for analyses. In youth ages 9 to 16 , the vertical jump has been shown to be reliable in both vertical concentric mean and peak power and eccentric mean power (Meylan, Cronin, Oliver, Hughes, \& McMaster, 2012).

\section{Procedures}

Data used in this study are drawn from the baseline data collected prior to the start of the REACH program (Late August and September 2016). Participants were provided with both the SQORD band and Actigraph devices prior to the start of the REACH program. Selected participants were reminded to wear both the Actigraph accelerometer and the SQORD band when given the devices and by the after-school teachers. There were no reminders given to students over the weekends. Those participants wearing the devices for at least four days during the data collection week had their names included in a drawing in which the winners (one girl and one boy) would receive a backpack. Upon return of the Actigraph accelerometers and SQORD bands one week later, study participants completed the battery of four fitness tests during a REACH program session.

\section{Data Analyses}

The data were screened for normality and a square root transformation was applied to nonnormally distributed variables to normalize the data. Descriptive statistics were calculated for all variables of interest using frequencies and proportions or means and standard deviations, as appropriate. Frequencies were used to show the proportion of participants with valid data based on combinations of hours of wear $(6,8$, and 10 hours) and days ( 1 day, 2 days, 3 , days, 4 days, 3 days excluding weekend days, and 4 days 
excluding weekend days) for both the SQORD band and the Actigraph accelerometer.

\begin{tabular}{|c|c|}
\hline \multicolumn{2}{|c|}{$\begin{array}{c}\text { Table 1. } \\
\text { Participant Characteristics }(N=41)\end{array}$} \\
\hline & $\mathrm{n}(\%)$ \\
\hline \multicolumn{2}{|l|}{ Grade } \\
\hline $5^{\text {th }}$ grade & $12(29.3)$ \\
\hline $6^{\text {th }}$ grade & $29(70.7)$ \\
\hline \multicolumn{2}{|l|}{ Sex } \\
\hline Male & $22(53.7)$ \\
\hline Female & $19(46.3)$ \\
\hline \multicolumn{2}{|l|}{ Ethnicity } \\
\hline Hispanic & $33(80.5)$ \\
\hline White & $3(7.3)$ \\
\hline Black & $2(4.9)$ \\
\hline Asian & $0(0.0)$ \\
\hline \multirow[t]{2}{*}{ Other } & $3(7.3)$ \\
\hline & Mean $\pm \mathrm{SD}, \mathrm{n}$ \\
\hline Age & $10.7 \pm 0.7,39$ \\
\hline Daily MVPA & \\
\hline Actigraph (min/day) & $36.5 \pm 14.9,17$ \\
\hline SQORD (pts/day) & $\begin{array}{r}85,652 \pm \\
19,640.4,38\end{array}$ \\
\hline $\begin{array}{l}\text { Muscular Endurance } \\
\text { Abdominal (\# curl-ups) }\end{array}$ & $30.5 \pm 22.0,36$ \\
\hline Upper Body (\# push-ups) & $10.9 \pm 7.7,36$ \\
\hline $\begin{array}{l}\text { Aerobic Capacity } \\
\text { PredictedVO } \\
\left(\mathrm{ml} \cdot \mathrm{kg}^{-1} \cdot \mathrm{min}^{-1}\right)\end{array}$ & $41.4 \pm 4.3,40$ \\
\hline $\begin{array}{l}\text { Leg Power } \\
\text { Vertical Jump (in.) }\end{array}$ & $11.5 \pm 3.0,33$ \\
\hline
\end{tabular}

The proportion of participants meeting of weartime compliance for 6,8 , and 10 hours were compared using the McNemar test for both the Actigraph accelerometer and the SQORD band. In order to examine if wear-time varied by activity levels, independent $t$-tests were used to analyze the difference in MVPA via Actigraph accelerometry and via the SQORD band between participants wearing the devices for 6 hours versus those who wore it for 10 hours.
The relationship between daily MVPA as measured by the SQORD band and the Actigraph accelerometer was assessed using bivariate Pearson correlation. Bivariate Pearson correlational analyses were further used to determine associations between the MVPA determined by Actigraph accelerometry and each fitness measure as well as between MVPA measured by the SQORD band and each fitness measure. All analyses were considered significant at a $p$-value of .05 .

\section{Results}

Forty-one youth participated in this study. Participant characteristics are presented in Table 1. The average daily MVPA ( $36.5 \pm 14.9$ minutes; via Actigraph) fell below the CDC recommended 60 minutes per day. The mean daily MVPA (via SQORD band) was $85651 \pm 19640$ points. Participants performed a mean of $30.5 \pm 22.0$ curl-ups and $10.9 \pm 7.7$ push-ups. The mean estimated $\mathrm{VO}_{2 \max }$ was $41.4 \pm 4.3 \mathrm{ml} \cdot \mathrm{kg}^{-1} \cdot \mathrm{min}^{-1}$ and the mean vertical jump height was $11.5 \pm 3.0$ inches.

\section{Wear-Time Compliance Sensitivity Analysis}

The sensitivity analysis of wear-time for 6,8 , and 10 hours for the Actigraph accelerometer and SQORD band is presented in Table 2. Just below $63 \%$ of participants met the valid wear-time criteria for 6 hours for the Actigraph accelerometer; this decreased to $22.2 \%$ at the criterion of 10 hours of wear-time. A significant difference was found in MVPA between those who wore the Actigraph accelerometer for 6 hours $(n=17,36.5 \pm 14.9 \mathrm{~min} /$ day $)$ versus those who wore it for 10 hours $(n=6,27.7 \pm 10.8$ $\min /$ day; $p=.024$ ).

For the SQORD band, approximately $66 \%$ of participants met the valid wear-time criteria for 6 hours while just $54 \%$ met the criteria of 10 hours of wear-time. A significantly greater proportion of participants met all criteria for wear-time when wearing the SQORD band in comparison to the Actigraph accelerometer (56.1\% versus $37.0 \%$, $\mathrm{p}=.004$ for 8 hours; $53.7 \%$, versus $22.2 \%, \mathrm{p}=.000$ for 10 hours). There were no significant differences between MVPA based on the SQORD for those who wore the SQORD for 6 
hours ( $n=27,85652 \pm 19640.4$ pts/day) versus those who wore it 10 hours $(n=22,89717 \pm$ $16679.9 ; p=.258$ ).

Table 2.

Frequency of Valid Wear-Time for 6, 8, and 10 Hours for the Actigraph and SQORD Actigraph Wear-Time Frequencies $(N=41)$

\begin{tabular}{ccc}
\hline Actigraph $(n=27)$ & $n(\%)$ \\
& 6 Hours & $17(62.9)$ \\
& 8 Hours & $10(37.0)$ \\
& 10 Hours & $6(22.2)$ \\
& Missing & $3(11.1)$ \\
\hline SQORD $(n=38)$ & & \\
& 6 Hours & $27(65.9)$ \\
& 8 Hours & $23(56.1)$ \\
& 10 Hours & $22(53.7)$ \\
Missing & $3(7.8)$ \\
\hline Both SQORD and Actigraph & \\
& 6 Hours & $17(41.5)$ \\
8 Hours & $10(24.4)$ \\
10 Hours & $6(14.6)$ \\
\hline
\end{tabular}

The proportion of participants meeting the valid wear-time based on a combination of hours of wear and days are presented in Table 3 . Thirtyseven percent of participants wore the Actigraph accelerometer for at least 8 hours for a minimum of three days, which decreased to $29.6 \%$ for at least four days. Participant compliance was lowest for all wear-time parameters requiring a minimum of 10 hours of wear-time (less than $23 \%$ ) for all day requirements. Over $58 \%$ of participants wore the SQORD band for a minimum for 4 days for at least 6,8 , and 10 hours per day.

\section{Associations Between MVPA (Actigraph), MVPA (SQORD), and Fitness Measures}

Daily MVPA measured by SQORD was moderately correlated with that measured via Actigraph accelerometry ( $r=0.651, p=.005)$. There were no significant correlations between MVPA (measured by either SQORD or Actigraph) and any measure of fitness $(r=-0.061$ $-0.817, p>.05$ for all; see Table 4$)$.

Table 3.

Percentage of Participants with Valid data for the Actigraph and SQORD Determined by Hours and Days of Wear $(\mathrm{N}=41)$

\begin{tabular}{lllllll}
\hline & 1 Day (\%) & 2 Day (\%) & 3 Day (\%) & 4 Day (\%) & $\begin{array}{l}\text { 3 Days- excluding } \\
\text { weekends (\%) }\end{array}$ & $\begin{array}{l}\text { 4 Day- excluding } \\
\text { weekends (\%) }\end{array}$ \\
\hline Actigraph $(n=27)$ & & & & & & \\
6 hours & 62.9 & 62.9 & 62.9 & 62.9 & 62.9 & 29.6 \\
8 hours & 37.0 & 37.0 & 37.0 & 29.6 & 33.3 & 33.3 \\
10 hours & 22.2 & 22.2 & 22.2 & 22.2 & 18.5 & 3.7 \\
\hline & & & & & & \\
SQORD $(n=38)$ & 80.5 & 75.6 & 65.9 & 65.9 & 58.5 & 56.1 \\
6 hours & 80.5 & 70.7 & 65.9 & 58.5 & 58.5 & 53.7 \\
8 hours & 80.5 & 68.3 & 65.9 & 58.5 & 56.1 & 51.2 \\
10 hours & & & & & & \\
\hline
\end{tabular}

Associations Between MVPA (Actigraph), MVPA (SQORD), and Fitness Measures Daily MVPA measured by SQORD was moderately correlated with that measured via Actigraph accelerometry ( $r=0.651, p=.005)$. There were no significant correlations between MVPA (measured by either SQORD or Actigraph) and any measure of fitness ( $r=-0.061$ $-0.817, p>.05$ for all; see Table 4).

\section{Discussion}

The purpose of this study was to assess freeliving MVPA and wear-time compliance between physical activity measured by a consumer-grade, wrist-worn activity monitor (SQORD band) and a research-grade, waist-worn accelerometer (Actigraph accelerometer) in low SES youth. We further aimed to better understand the SQORD band by examining the relationships between daily MVPA collected by both devices and various fitness measures. 
Table 4.

Correlation of MVPA with Fitness Measures

\begin{tabular}{lll}
\hline & Pearson $r$ & $p$ \\
\hline MVPA via Actigraph & & \\
Curl-Up ( $n=14)$ & 0.425 & 0.129 \\
$90^{\circ}$ Push-Up $(n=14)$ & 0.506 & 0.065 \\
PACER Estimated & 0.339 & 0.199 \\
VO2max ( $n=16)$ & & \\
Vertical Jump $(n=17)$ & -0.061 & 0.817 \\
\hline MVPA via SQORD & & \\
Curl-Up ( $n=21)$ & 0.315 & 0.165 \\
90 Push-Up $(n=21)$ & 0.338 & 0.134 \\
PACER Estimated & 0.111 & 0.524 \\
VO2max $(n=22)$ & & \\
Vertical Jump $(n=23)$ & 0.107 & 0.627 \\
\hline
\end{tabular}

Fewer than $23 \%$ of youth met the standard weartime compliance of 10 hours per day on four days for the Actigraph accelerometer, and only around $54 \%$ met the same criteria for the SQORD band. It is possible that the sample was biased, perhaps underestimating actual use among those youth who were particularly inactive. However, sensitivity analyses showed participants wearing the Actigraph accelerometer for 6 hours per day participated in daily MVPA significantly more than those who met the standard 10 hours per day criterion. In general, the objective measurement of MVPA among children and youth is a challenging endeavor, with studies suggesting that reactivity (change in normal behavior) and tampering influence the data (Scott, Morgan, Plotnikoff, Trost, \& Lubans, 2014).

The location of the Actigraph accelerometer on the waist during data collection may provide another explanation for the low wear-time compliance, as discussed by Scott et al. (2017). Their study suggested that wrist-worn devices are preferred because they are likely to be more comfortable and less embarrassing. Furthermore, youth in the study reported a non-preference for waist-worn accelerometers and the authors reported a high incidence (approximately 25\% of days) of missing data in their cohort (Scott et al., 2017). In the present study, wear-time compliance was significantly higher for the wristworn consumer device compared to the waist- worn accelerometer, in agreement with studies by Scott et al. and others (Fairclough et al., 2016; Scott et al., 2017). It is possible that the SQORD band was preferred over the Actigraph accelerometer for device design reasons, including the SQORD band providing simple feedback and connecting the user to a game and the waterproof nature of the band eliminating the need for participants to remove it; however this question was not addressed within our study. Nonetheless, the wrist-worn band appears to be a convenient consumer-grade activity tracker for youth possibly due to its wrist-worn capabilities and its fun-oriented design.

A novel aspect of the current study is the analyses of accelerometer wear-time adherence in a lowSES, mostly Hispanic population. A study by Evenson and colleagues (2015) directly analyzed wear-time adherence, finding that approximately $92 \%$ of a large cohort (more than 16,000 participants) of Hispanic adults had at least one day of valid wear-time and $77.7 \%$ adhering to the wear-time parameters of at least 3 days. Ruiz et al. (2011) studied the relationship between Hispanic parents' and children's physical activity patterns and found that, out of 106 parent-child dyads, $75.4 \%$ of children and $80.2 \%$ of parents had usable accelerometry data. However, analyses by Schaefer and colleagues (2016) found low engagement (wearing and syncing the activity device regularly) in a group of urban youth. This study found low accelerometer weartime compliance in our mostly Hispanic sample, with only $62.9 \%$ of participants meeting the 6 hours per day wear-time criterion for only one day. Evidence suggests careful monitoring and consistent encouragement from research staff for wear-time adherence in youth is essential, so it is possible that greater monitoring and encouragement from research staff could have improved wear-time compliance in our young participants (Schaefer et al., 2016; Van Coevering et al., 2005).

The primary purpose of this study was to correlate the SQORD activity monitor against the Actigraph GT3X accelerometer. Results indicate that there was a moderate correlation between the two devices in the measurement of MVPA. This finding agrees with the study by Sirard et al. 
(2017) which demonstrated the SQORD band was consistently sufficient in measuring both structured and free-living physical activity and exhibited higher and more stable day-to-day correlations compared to the Actigraph accelerometer. These findings further corroborate that the SQORD band is able to measure freeliving MVPA, which is especially important due to the increased popularity of consumer-grade activity trackers. The findings suggest that the SQORD band may be useful in future interventions in which direct, timely MVPA feedback to youth is desired, given that the participants in this study were much more compliant in wearing the consumer-grade device compared to the research-grade device.

There were no significant associations between the daily MVPA collected either via SQORD band or Actigraph and the various fitness measures. Other studies have demonstrated that higher daily physical activity is associated with higher fitness levels in youth (Aires et al., 2010; Lohman et al., 2008; Martinez-Gomez et al., 2011). The lack of significant associations in this study may be due to the relatively low sample size obtained because the participants did not wear the devices very often or for very long, resulting in many participants with missing MVPA data. Furthermore, due to lack of permission from the school board, pubertal status was not assessed in this study, which is associated with fitness level in both sexes (Batista et al., 2017; Gammon, Pfeiffer, Kazanis, Ling, \& Robbins, 2017). Associations between physical activity and various fitness measures may have been more evident had we controlled for maturation.

\section{Limitations}

In addition to the limitations outlined above, it is possible that the wear-time between the devices may not have occurred during the same 6-hour period on a given day. While recognizing that this may be a limitation in our data, we were primarily interested in average daily MVPA, so the time period in which the activity was accrued was not of primary interest. Our study highlights the difficulty of measuring physical activity in children and youth, as demonstrated by our low wear-time compliance. Previous studies have also found this to be a challenge. As outlined earlier,
Scott et al. (2014) found low wear-time compliance among youth participants, particularly with waist-worn devices along with evidence of reactivity and device tampering. Another recent study found approximately $31 \%$ of boys and girls did not meet wear-time compliance when measuring physical activity using an arm-worn (worn on the upper arm/triceps area) accelerometer (Saint-Maurice, Kim, \& Welk, 2017). We attempted to address the challenge of low wear-time compliance by providing an incentive to those who met certain wear-time parameters. Other studies have provided incentives, such as Scott et al. (2014) who provided gift bags to those who returned the pedometer. However, low wear-time compliance remained an issue in these studies. Tudor-Locke et al. (2015) found youth 9 to 11 years had higher wear-time compliance when asked to wear the waist-worn accelerometer for 24-hours during the duration of the collection period compared to youth who were asked to wear it only during waking hours. Thus, altering accelerometer data collection protocols may result in higher weartime compliance in youth, but more research would need to confirm this speculation.

\section{Conclusion}

This is only the second study to examine the SQORD band in monitoring free-living MVPA among youth. Novel analyses in the present study include the comparisons of wear-time between an expensive, research-grade accelerometer and a cost- and user-friendly activity tracker in a low SES, mostly Hispanic youth population. Due to its ability to effectively measure MVPA in elementary-aged youth, the SQORD band appears to be a cost-friendly and feasible device to measure daily MVPA for youth and could be a possible option for tracking MVPA for lowbudget research studies. Groups who are interested in tracking the physical activity of youth, such as after-school programs, physical education programs, teachers, and researchers, should consider using wrist-worn, consumergrade devices, as youth appear to favor it over waist-worn, research-grade devices. In order to be effective, however, future research will need to consider strategies to increase wear-time compliance in youth study populations. 


\section{References}

Actigraph Corporation (2017). Actigraph GT9X link. Retrieved from https://www.actigraphcorp.com/actigraph-link/

Aires, L., Silva, P., Silva, G., Santos, M. P., Ribeiro, J. C., \& Mota, J. (2010). Intensity of physical activity, cardiorespiratory fitness, and body mass index in youth. Journal of Physical Activity and Health, 7(1), 54-59.

Andersen, L. B., Harro, M., Sardinha, L. B., Froberg, K., Ekelund, U., Brage, S., \& Anderssen, S. A. (2006). Physical activity and clustered cardiovascular risk in children: A cross-sectional study (The European Youth Heart Study). Lancet, 368(9532), 299-304. doi:10.1016/S01406736(06)69075-2

Anderson, E. A., Zhang, J. J., Rudisill, M. E., \& Gaa, J. (1997). Validity and reliability of a timed curl-up test: Development of a parallel form for the FITNESSGRAM abdominal strength test. Research Quarterly for Exercise and Sport, 68(Suppl), A-51.

Basch, C. E. (2011). Physical activity and the achievement gap among urban minority youth. Journal of School Health, 81(10), 626-634. doi:10.1111/j.1746-1561.2011.00637.x

Batista, M. B., Valente-Dos-Santos, J., Duarte, J. P., Sousa, E. S. P., Coelho, E. S. M. J., Werneck, A. O., ... Ronque, E. R. V. (2017). Independent and combined effects of weight status and maturation on aerobic fitness in adolescent school aged males. Journal of Strength \& Conditioning Research. doi:10.1519/JSC.0000000000002363

Brodersen, N. H., Steptoe, A., Boniface, D. R., \& Wardle, J. (2007). Trends in physical activity and sedentary behaviour in adolescence: Ethnic and socioeconomic differences. British Journal of Sports Medicine, 41(3), 140-144. doi:10.1136/bjsm.2006.031138

Case, M. A., Burwick, H. A., Volpp, K. G., \& Patel, M. S. (2015). Accuracy of smartphone applications and wearable devices for tracking physical activity data. Journal of the American Medical Association, 313(6), 625-626. doi:10.1001/jama.2014.17841

Centers for Disease Control (CDC) (2017). Healthy Schools. Retrieved from https://www.cdc.gov/healthyschools/physicalactivity/guidelines.htm

Chu, A. H., Ng, S. H., Paknezhad, M., Gauterin, A., Koh, D., Brown, M. S., \& Müller-Riemenschneider, F. (2017). Comparison of wrist-worn Fitbit Flex and waist-worn ActiGraph for measuring steps in free-living adults. Public Library of Science One, 12(2), e0172535. doi:10.1371/journal.pone.0172535

DiStefano, L. J., Padua, D. A., Blackburn, J. T., Garrett, W. E., Guskiewicz, K. M., \& Marshall, S. W. (2010). Integrated injury prevention program improves balance and vertical jump height in children. Journal of Strength \& Conditioning Research, 24(2), 332-342. doi:10.1519/JSC.0b013e3181cc2225

Dominick, G. M., Winfree, K. N., Pohlig, R. T., \& Papas, M. A. (2016). Physical activity assessment between consumer- and research-grade accelerometers: A comparative study in free-living conditions. Journal of Medical Internet Research mHealth uHealth, 4(3), e110. doi:10.2196/mhealth.6281

Education Data Partnership (2017). School profile. Retrieved from https://www.ed-data.org/school

Evenson, K. R., Catellier, D. J., Gill, K., Ondrak, K. S., \& McMurray, R. G. (2008). Calibration of two objective measures of physical activity for children. Journal Sports Science, 26(14), 1557-1565. doi:10.1080/02640410802334196

Evenson, K. R., Goto, M. M., \& Furberg, R. D. (2015). Systematic review of the validity and reliability of consumer-wearable activity trackers. International Journal of Behavioral Nutrition and Physical Activity, 12, 159. doi:10.1186/s12966-015-0314-1

Evenson, K. R., Sotres-Alvarez, D., Deng, Y. U., Marshall, S. J., Isasi, C. R., Esliger, D. W., \& Davis, S. (2015). Accelerometer adherence and performance in a cohort study of US Hispanic adults. Medicine \& Science in Sports \& Exercise, 47(4), 725-734. doi:10.1249/MSS.0000000000000478 
Fairclough, S. J., Noonan, R., Rowlands, A. V., Van Hees, V., Knowles, Z., \& Boddy, L. M. (2016). Wear compliance and activity in children wearing wrist- and hip-mounted accelerometers. Medicine \& Science in Sports \& Exercise, 48(2), 245-253. doi:10.1249/MSS.0000000000000771

Fanning, J., Mullen, S. P., \& McAuley, E. (2012). Increasing physical activity with mobile devices: A meta-analysis. Journal of Medical Internet Research, 14(6), e161. doi:10.2196/jmir.2171

Ferguson, T., Rowlands, A. V., Olds, T., \& Maher, C. (2015). The validity of consumer-level, activity monitors in healthy adults worn in free-living conditions: A cross-sectional study. International Journal of Behavioral Nutrition and Physical Activity, 12, 42. doi:10.1186/s12966-015-0201-9

Frederick, C. B., Snellman, K., \& Putnam, R. D. (2014). Increasing socioeconomic disparities in adolescent obesity. Proceedings of the National Academy of Sciences of the United States of America, 111(4), 1338-1342. doi:10.1073/pnas.1321355110

Gammon, C., Pfeiffer, K. A., Kazanis, A., Ling, J., \& Robbins, L. B. (2017). Cardiorespiratory fitness in urban adolescent girls: Associations with race and pubertal status. Journal of Sports Science, 35(1), 29-34. doi:10.1080/02640414.2016.1154594

Garde, A., Umedaly, A., Abulnaga, S. M., Robertson, L., Junker, A., Chanoine, J. P., . . Dumont, G. A. (2015). Assessment of a mobile game ("MobileKids Monster Manor") to promote physical activity among children. Games for Health Journal, 4(2), 149-158. doi:10.1089/g4h.2014.0095

Hänggi, J. M., Phillips, L. R., \& Rowlands, A. V. (2013). Validation of the GT3X ActiGraph in children and comparison with the GT1M ActiGraph. Journal of Science and Medicine in Sport, 16(1), 4044. doi:10.1016/j.jsams.2012.05.012

Katzmarzyk, P. T., Denstel, K. D., Beals, K., Bolling, C., Wright, C., Crouter, S. E., . . Sisson, S. B. (2016). Results from the United States of America's 2016 Report Card on Physical Activity for Children and Youth. Journal of Physical Activity and Health, 13(11 Suppl 2), S307-S313. doi:10.1123/jpah.2016-0321

Lee, J. A., Williams, S. M., Brown, D. D., \& Laurson, K. R. (2014). Concurrent validation of the Actigraph gt3x+, Polar Active accelerometer, Omron HJ-720 and Yamax Digiwalker SW-701 pedometer step counts in lab-based and free-living settings. Journal of Sports Science, 33(10), 991-1000. doi:10.1080/02640414.2014.981848

Leger, L. A., \& Gadoury, C. (1989). Validity of the $20 \mathrm{~m}$ shuttle run test with 1 min stages to predict VO2max in adults. Canadian Journal of Sport Sciences, 14, 21-26.

Leger, L. A., \& Lambert, J. (1982). A maximal multistage 20-m shuttle run test to predict VO2max. European Journal of Applied Physiology and Occupational Physiology, 49, 1-12.

Leger, L. A., Mercier, D., Gadoury, C., \& Lambert, J. (1988). The multistage 20 metre shuttle run test for aerobic fitness. Journal of Sports Sciences, 6, 93-101.

Lohman, T. G., Ring, K., Pfeiffer, K., Camhi, S., Arredondo, E., Pratt, C., .. W Webber, L. S. (2008). Relationships among fitness, body composition, and physical activity. Medicine \& Science in Sports \& Exercise, 40(6), 1163-1170. doi:10.1249/MSS.0b013e318165c86b

Mahar, M. T., Guerieri, A. M., Hanna, M. S., \& Kemble, C. D. (2011). Estimation of aerobic fitness from 20-m multistage shuttle run test performance. American Journal of Preventive Medicine, 41(4 Suppl 2), S117-123. doi:10.1016/j.amepre.2011.07.008

Martinez-Gomez, D., Welk, G. J., Puertollano, M. A., Del-Campo, J., Moya, J. M., Marcos, A., . . . Group, A. S. (2011). Associations of physical activity with muscular fitness in adolescents. Scandinavian Journal of Medicine \& Science in Sports, 21(2), 310-317. doi:10.1111/j.16000838.2009.01036.x

Marttinen, R., Fredrick, R., Johnston, K., Phillips, S., \& Patterson, D. (2019) Implementing the REACH after-school program for youth in urban communities: Challenges and lessons learned. European Physical Education Review. (On-line first) https://doi.org/10.1177/1356336X19865566

Marttinen, R., \& Fredrick. R. (2017). R.E.A.C.H: A case for after school physical education. Strategies, $30(1), 8-14$. 
Masteller, B., Sirard, J., \& Freedson, P. (2017). The Physical Activity Tracker Testing in Youth (P.A.T.T.Y.) Study: Content analysis and children's perceptions. Journal of Medical Internet Research mHealth uHealth, 5(4), e55. doi:10.2196/mhealth.6347

Meylan, C. M., Cronin, J. B., Oliver, J. L., Hughes, M. G., \& McMaster, D. T. (2012). The reliability of jump kinematics and kinetics in children of different maturity status. Journal of Strength and Conditioning Research, 26(4), 1015-1026. doi:10.1519/JSC.0b013e31822dcec7

Paliczka, V. J., Nichols, A. L., \& Boreham, A. G. (1987). A multi-stage shuttle run as a predictor of running performance and maximal oxygen uptake in adults. British Journal of Sports Medicine, 21, 163-165.

Patterson, P., Rethwisch, N., \& Wiksten, D. (1997). Reliability of the trunk lift in high school boys and girls. Measurement of Physical Education and Exercise Science, 1, 145-151.

Plowman, S. A. (2013). Muscular Strength, Endurance, and Flexibility Assessments. In Fitnessgram/Activitygram Reference Guide (4th ed., pp. Internet Resource). Dallas, TX: The Cooper Institute.

Ramsbottom, R., Brewer, J., \& Williams, C. (1988). A progressive shuttle run test to estimate maximal oxygen uptake. British Journal of Sports Medicine, 22, 141-144.

Rich, C., Geraci, M., Griffiths, L., Sera, F., Dezateux, C., \& Cortina-Borja, M. (2013). Quality control methods in accelerometer data processing: Defining minimum wear time. Public Library of Science One, 8(6), e67206. doi:10.1371/journal.pone.0067206

Ruiz, R., Gesell, S. B., Buchowski, M. S., Lambert, W., \& Barkin, S. L. (2011). The relationship between Hispanic parents and their preschool-aged children's physical activity. Pediatrics, 127(5), 888895. doi:10.1542/peds.2010-1712

Saint Romain, B., \& Mahar, M. T. (2001). Norm-referenced and critierion-referenced reliability of the push-up and modified pull-up. Measurement of Physical Education and Exercise Science, 5, 6780.

Saint-Maurice, P. F., Kim, Y., \& Welk, G. J. (2017). Evidence for data missing at random in youth physical activity monitoring research. Journal of Sports Science, 35(5), 484-490. doi:10.1080/02640414.2016.1173719

Schaefer, S. E., Ching, C. C., Breen, H., \& German, J. B. (2016). Wearing, thinking, and moving: testing the feasibility of fitness tracking with urban youth. American Journal of Health Education, 47(1), 8-16. doi:10.1080/19325037.2015.1111174

Scott, J. J., Morgan, P. J., Plotnikoff, R. C., Trost, S. G., \& Lubans, D. R. (2014). Adolescent pedometer protocols: Examining reactivity, tampering and participants' perceptions. Journal of Sports Science, 32(2), 183-190. doi:10.1080/02640414.2013.815361

Scott, J. J., Rowlands, A. V., Cliff, D. P., Morgan, P. J., Plotnikoff, R. C., \& Lubans, D. R. (2017). Comparability and feasibility of wrist- and hip-worn accelerometers in free-living adolescents. Journal of Science and Medicine in Sport. doi:10.1016/j.jsams.2017.04.017

Sirard, J. R., Masteller, B., Freedson, P. S., Mendoza, A., \& Hickey, A. (2017). Youth oriented activity trackers: Comprehensive laboratory- and field-based validation. Journal of Medical Internet Research, 19(7), e250. doi:10.2196/jmir.6360

SQORD (2015). Team Admin's Playbook. Durham, NC.

Stalsberg, R., \& Pedersen, A. V. (2010). Effects of socioeconomic status on the physical activity in adolescents: A systematic review of the evidence. Scandinavian Journal of Medicine \& Science in Sports, 20(3), 368-383. doi:10.1111/j.1600-0838.2009.01047.x

Tudor-Locke, C., Barreira, T. V., Schuna, J. M., Jr., Mire, E. F., Chaput, J. P., Fogelholm, M., . . Group, I. R. (2015). Improving wear time compliance with a 24-hour waist-worn accelerometer protocol in the International Study of Childhood Obesity, Lifestyle and the Environment (ISCOLE). International Journal of Behavioral Nutrition and Physical Activity, 12, 11. doi:10.1186/s12966015-0172-x 
Tully, M. A., McBride, C., Heron, L., \& Hunter, R. F. (2014). The validation of Fibit Zip ${ }^{\mathrm{TM}}$ physical activity monitor as a measure of free-living physical activity. Biomed Central Research Notes, 7, 952. doi:10.1186/1756-0500-7-952

Van Coevering, P., Harnack, L., Schmitz, K., Fulton, J. E., Galuska, D. A., \& Gao, S. (2005). Feasibility of using accelerometers to measure physical activity in young adolescents. Biomed Central Research Notes, 37(5). doi:10.1186/1756-0500-7-952

Corresponding Author Information

Risto Marttinen (ORCID: 0000-0003-3807-5684)

Assistant Professor

George Mason University

10900 George Mason Circle, MS 4E5, Katherine G. Johnson

Hall, Manassas, VA, 20110

rmarttin@gmu.edu 